\title{
RADIOGRAFÍA DE LOS MODELOS DE ACREDITACIÓN: ORGANIZACIÓN, PROCESOS Y PRÁCTICAS. EL CASO DE LAS UNIVERSIDADES AUSTRAL DE CHILE, DE LA FRONTERA Y DE LOS LAGOS $^{1}$
}

\author{
Juan Pablo Venables ${ }^{2}$, Jilles Van Gastel ${ }^{3}$
}

\begin{abstract}
RESUMEN
La instauración de políticas de aseguramiento de la calidad en Chile ha traído aparejada el desarrollo, por parte de cada institución, de sistemas y mecanismos propios para abordarlas. No obstante, existe una carencia de reflexión sistémica al respecto, que permita utilizar estas experiencias como instancias de aprendizaje para el sistema en su conjunto, más allá de las fronteras de cada institución. Utilizando un enfoque sociológico, este artículo intenta comprender, exponer y analizar a través de una investigación comparada, los distintos "modos de organización" de los procesos de acreditación institucional desarrollados por las universidades Austral de Chile, de La Frontera y de Los Lagos, dando cuenta de la multiplicidad de "modos de organización" existentes y poniendo énfasis en que esta diversidad se explica al comprender la internalización de la Ley N²0.129 por parte de las IES como un proceso de "traducción" y "significación". Como resultado, se relevan particularidades de cada caso y experiencias compartidas, al mismo tiempo que se identifican buenas prácticas y desafíos, tanto para estas universidades en particular, como para las IES en su conjunto.
\end{abstract}

Palabras clave: acreditación, Ley 20.129, aseguramiento de la calidad, autoevaluación, autorregulación.

\section{A SNAPSHOT OF ACCREDITATION MODELS: ORGANIZATION, PROCESSES AND PRACTICES. THE CASE OF THE UNIVERSITIES AUSTRAL OF CHILE, FRONTERA AND LAGOS}

\section{ABSTRACI}

The establishment of quality assurance policies in Chile has necessitated the development of systems and mechanisms to address them. Institutions have created their own mechanisms, however, revealing that a systematic approach is missing that would enable these experiences to transform into learning opportunities for the system as a whole, beyond the enclosed walls of each institution. Utilizing a sociological approach, this article aims to understand, present and analyze by way of comparative research, the distinct "organization methods" for the institutional accreditation processes developed by the Universities Austral of Chile, Frontera and Lagos. This article will attempt to reveal the variation in existing "organization methods" and emphasize that this diversity can be explained by understanding the internalization of the Legislation $N^{\circ} 20.129$ by the Institutions of Higher Learning as a process of "translation" and "significance". In conclusion, particularities of each case and experiences in common will be presented, along with the identification of good practices and ongoing challenges, as much for these universities in particular, as for the world of Institutions of Higher Learning collectively.

Keywords: accreditation, Legislation 20.129, quality assurance, self-assessment, self-regulation.

1 El presente artículo resume los aspectos principales y los resultados más destacados del proyecto de investigación que lleva el mismo nombre, desarrollado durante los años 2013 2014 en el marco de la Convocatoria de apoyo a la investigación en temas de educación del Consejo Nacional de Educación.

2 Instituto de Historia y Ciencias Sociales, Facultad de Filosofía y Humanidades, Universidad Austral de Chile, Valdivia, Chile. Contacto: jpvenables@uach.cl

3 Instituto de Administración, Facultad de Ciencias Económicas y Administrativas, Universidad Austral de Chile, Valdivia, Chile. Contacto: jilles.vangastel@uach.cl 
52 RADIOGRAFÍA DE LOS MODELOS DE ACREDITACIÓN: ORGANIZACIÓN, PROCESOS Y PRÁCTICAS. EL CASO DE LAS UNIVERSIDADES AUSTRAL DE CHILE, DE LA FRONTERA Y DE LOS LAGOS - J. P. Venables, J. Van Gastel

\section{Introducción}

El fortalecimiento de la calidad de la educación superior en Chile es un tema que está presente tanto en el debate nacional como en la orientación de las políticas públicas en educación desde hace más de una década. En este contexto, el año 2006 se dictó la Ley 20.129, que establece un Sistema Nacional de Aseguramiento de la Calidad, cuya finalidad es integrar las funciones de información, licenciamiento, acreditación institucional y de programas que se encontraban disgregadas en distintos organismos y sin un marco normativo común.

Uno de los ejes principales de este sistema es la acreditación institucional, que ha enfrentado a las instituciones de educación superior (IES) y en particular a las universidades, con el desafío de incorporar en las distintas áreas de su quehacer institucional mecanismos de autoevaluación, autorregulación institucional y mejoramiento continuo.

Si bien el Sistema Nacional de Aseguramiento de la Calidad establece un proceso de acreditación único para las distintas instituciones, cada una debe resolver internamente cómo lleva adelante el proceso de autoevaluación, cómo hace el seguimiento de los planes de mejora y, en definitiva, cómo gestiona la acreditación institucional e incorpora mecanismos de mejoramiento continuo. A modo referencial, para el año 2013, de las 25 universidades que componen el Consejo de Rectores de las Universidades Chilenas (CRUCH), 20 de ellas cuentan con direcciones superiores que llevan adelante estos procesos, mientras que el resto posee unidades, oficinas o departamentos que cumplen con esta finalidad. De estas 20 direcciones, algunas dependen de Rectoría, otras de la Vicerrectoría de planificación y otras de la Vicerrectoría académica, lo que más allá de la diferencia en la estructura organizacional, implica funciones diversas relacionadas con la planificación estratégica, el análisis institucional, la provisión de información para la toma de decisiones, el seguimiento del plan estratégico y la orientación del proceso de autoevaluación, entre otras.

En este contexto, todo parece indicar que la decisión acerca de cómo incorporar mecanismos de aseguramiento de la calidad y, más 
específicamente, cómo organizar los procesos de acreditación por parte de cada institución, se resuelve de manera interna y en función de variables netamente institucionales. En consecuencia, se puede sostener que cada institución ha desarrollado sus propios diseños y prácticas, que traen como resultado que bajo la unidad normativa del sistema de acreditación, exista una "multiplicidad de modelos" de organización entre las instituciones.

Como correlato de esta multiplicidad de modelos de gestión de la acreditación se encuentra el carácter endogámico en que éstos se desarrollan. En efecto, las distintas experiencias y aprendizajes derivados de este proceso difícilmente han superado las fronteras institucionales, restringiéndose endogámicamente a la comparación de las instituciones consigo mismas y limitando las reflexiones e investigaciones a una comparación de sus resultados, expresados en el número de años de acreditación. En consecuencia, existe una carencia de reflexión sistémica al respecto, que permita utilizar estos distintos modelos y prácticas como instancias de aprendizaje para el sistema en su conjunto, más allá de las fronteras de cada institución.

En ese contexto, este trabajo realiza una investigación comparada de los "modos de organización"4 de los procesos de acreditación desarrollados por la Universidad Austral de Chile (UACh), la Universidad de La Frontera (UFRO) y la Universidad de Los Lagos (ULagos). Su elección radica -entre otras cosas- en que representan casos explícitos de esta diversidad de "modos de organización" ". Asimismo, los resultados de acreditación obtenidos por estas tres instituciones son dispares. A nivel institucional, la UACh está acreditada por seis años en todas las áreas (2009-2015), la UFRO por cinco años en todas las áreas (2013-2018) y la ULagos por tres años en las áreas de Gestión Institucional, Docencia de Pregrado y Vinculación con el Medio (2013-2016).

4 Con "modo de organización" se hace referencia a un concepto teórico que reúne dos aspectos: el modelo (aspectos formales) y las prácticas (aspectos informales). Esta idea se desarrolla más profundamente en el apartado metodológico.

5 La elección de estos casos se explica en el marco metodológico. 
Si bien dichos resultados no dependen enteramente de cómo se organizan los procesos de autoevaluación y de mejoramiento continuo, no es posible obviar la importancia que tiene en el proceso mismo el "modo de organización" de acreditación utilizado por cada universidad.

Dicho lo anterior, el objetivo general de la investigación es comprender, exponer y analizar a través de una investigación comparada, los distintos "modos de organización" de los procesos de acreditación institucional desarrollados por las universidades Austral de Chile, de La Frontera y de Los Lagos.

\section{Estado del arte y contexto del problema}

La entrada en vigencia del Sistema Nacional de Aseguramiento de la Calidad en el año 2006 trajo aparejada cambios tanto al interior de las instituciones como en el sistema en su conjunto, principalmente en lo que se refiere a la formalización de los procesos de acreditación -sean estos institucionales o de carreras/programas-, tal como se refleja en la definición de dos de las cuatro funciones que la ley le confiere ${ }^{6}$.

Pero, junto con la importancia legal que los procesos de acreditación tienen dentro de este sistema, se encuentra la relevancia que las mismas IES les otorgan. Estas califican la acreditación como "un proceso de carácter fiscalizador muy necesario y pertinente para el adecuado funcionamiento del sistema de educación superior" (IPSOSb, 2010, p. 11). En consecuencia, una gran mayoría considera que "es un proceso que instala una mayor preocupación por el aseguramiento de la calidad" (IPSOSb, 2010, p. 11), fortaleciendo la capacidad de autorregulación, permitiendo una mayor formalización de procedimientos y responsabilidades y generando mejoras a nivel de planificación y gestión de las IES.

El actual proceso de acreditación institucional y de programas se divide y sustenta en tres etapas principales: 1) autoevaluación, realizada por la institución y/o programa; 2) evaluación externa

6 Artículo $1^{\circ}$ Ley $N^{\circ} 20.129$, que establece un Sistema Nacional de Aseguramiento de la Calidad de la Educación Superior. 
(comité de pares); y 3) evaluación y posterior decisión de acreditación por parte de la CNA o de la agencia. Pese a la importancia de cada una de estas tres etapas, la mayor parte de las reflexiones y discusiones públicas en torno al sistema de acreditación se ha centrado principalmente en la tercera y, en menor medida en la segunda, dejando la etapa de autoevaluación -y con ello a los sistemas de gestión institucionales que la sustentan- como un asunto interno de cada institución, que se resuelve de manera endogámica y en función de variables netamente institucionales.

En efecto, como señala Lemaitre al respecto:

"En nuestro país no existen aún suficientes estudios sistemáticos que den cuenta de los efectos que ha tenido la acreditación en instituciones de educación superior (IES), por lo que no hay evidencia disponible que identifique mecanismos o prácticas que favorezcan una eficiente gestión de la calidad al interior de las instituciones" (Lemaitre, Maturana, Zenteno y Alvarado, 2012, p. 22).

En consecuencia, si bien el principal objetivo de la acreditación es fomentar una cultura de autorregulación al interior de las instituciones de educación superior (primera etapa), como se dijo, las distintas experiencias y aprendizajes derivados de este proceso no han generado reflexiones sistemáticas que constituyan un aporte al sistema en su conjunto, toda vez que se han considerado temas internos de cada institución.

\section{Marco teórico}

Esta investigación propone un enfoque sociológico a través del cual se concibe el proceso de instalación en las universidades de la política pública expresada en la Ley No 20.129 como un proceso de "traducción" (Latour, 1996) y, por tanto, de generación de "significados" (Yanow, 2013). Es decir, se entiende la multiplicidad de "modos de organización" del aseguramiento de la calidad como el efecto de un proceso de "traducción" por parte de las IES, más que de "implementación” de una política pública unívoca (Latour, 1996), lo que ha traído como consecuencia que la apropiación e internalización 
de esta demanda externa por parte de cada universidad haya generado distintos "significados" en torno a una normativa común.

Ambos conceptos -traducción y significación-implícitamente incorporan una perspectiva situada, destacando lo que es particular de cada universidad (Koselleck, 1993).

Esta "significación" se estudia por medio de dos vías complementarias: la primera dice relación con el estudio comparativo de las unidades encargadas de llevar adelante estos procesos de "significación" al interior de las universidades. En efecto, una de las consecuencias más visibles de la implementación del Sistema Nacional de Aseguramiento de la Calidad y de los procesos de acreditación en particular es la creación, por parte de las universidades, de unidades especializadas de llevar adelante esta tarea.

En vinculación con la primera, la segunda vía hace referencia al sistema -en abstracto- creado por cada universidad para desarrollar los procesos de autoevaluación institucional y de seguimiento de la planificación de mejoras derivados de los primeros, que para efectos metodológicos y conceptuales se los concibe como "modos de organización" de los procesos de acreditación.

A su vez, dichos modos están conformados por dos aspectos disociables sólo teóricamente: modelos y prácticas. Los modelos se comprenden por:

- la trayectoria formal de la información relativa a los procesos de acreditación escrita en documentos

- la división oficial de roles, responsabilidades y funciones de las distintas unidades encargadas de dichos procesos

- las relaciones oficialmente establecidas entre las distintas instancias vinculadas

- las reglas y los procedimientos oficiales que conforman el modelo de organización de la acreditación

Pero la organización de estos procesos no consiste sólo en el diseño formal u oficial de los modelos de acreditación, sino también se compone de prácticas organizativas. La teoría de las prácticas 
opone a la idea de que son las reglas y los procedimientos explícitos los que dirigen la actividad social, la concepción de que, en su lugar, las prácticas son las generadoras de fenómenos sociales (Schatzki, 2001). En este sentido, entendemos las prácticas como colecciones de acciones humanas, conocimiento tácito y aprendizaje compartido que, a la vez que dan sustento a cualquier cuerpo normativo o procedimental, los trascienden (Schatzki, 2001).

En definitiva, las universidades han concentrado el trabajo de "traducción" y "significación" en unidades especializadas que tienen como tarea principal llevar adelante los procesos de acreditación al interior de cada institución; tarea que hacen por medio del desarrollo conjunto de un modelo de organización entendido como reglamentos y procedimientos formalizados- y de prácticas organizativas -reconocidas como aspectos informalesque, globalmente, comprendemos como el "modo de organización" particular de cada institución.

El análisis, tanto de las unidades ejecutoras como de los "modos de organización", se desarrolla en función de cuatro categorías que rescatan los principales aspectos organizacionales vinculados con estos procesos. El contenido teórico y conceptual de cada una de estas categorías se expone en el cuerpo del análisis.

\section{Marco metodológico}

Dado que el objetivo principal de esta investigación es de tipo cualitativo, esta se lleva a cabo a través de la metodología de estudio de casos comparados o múltiples (Yin, 1994). Esta metodología permite, desde situaciones empíricas particulares, extraer lecciones generales en torno a la implementación de la Ley No 20.129, la creación de modelos de autoevaluación y aseguramiento de la calidad, e indagar acerca de cómo se han tomado decisiones y resuelto dificultades y desafíos que son consustanciales a estas políticas de monitoreo y evaluación.

Para la producción de información se han usado tres técnicas: la primera es la revisión documental, mayormente a través de decretos y resoluciones, informes de autoevaluación institucional y planes 
estratégicos. La segunda técnica son las entrevistas en profundidad, cara a cara, semiestructuradas y con preguntas abiertas. Para ello, en cada universidad se entrevistó al primer encargado de la unidad de aseguramiento de la calidad, al encargado actual de la unidad, a un funcionario de la misma unidad, a un académico con participación en el último proceso de acreditación institucional, al rector, y en los casos de la Universidad Austral de Chile y la Universidad de Los Lagos, a un representante de alguna sede. La tercera técnica es la observación, enfocada principalmente en el ambiente o espacio físico con que cuentan estas unidades. Las entrevistas y la observación se llevaron a cabo entre abril y junio de 2014.

Las tres técnicas generaron información que, desde una perspectiva constructivista o simbólica, se concibió y clasificó como información factual. Es decir, para el análisis se puso énfasis en los "hechos", entendidos como fechas y eventos compartidos o al menos no cuestionados por otra fuente de información (Law, 1994), lo que no quita que también se destaquen ciertos disensos en aspectos que así lo ameritan.

Por otra parte, los documentos, las entrevistas y la observación, también producen información a nivel simbólico (Yanow, 2013). En consecuencia, en la comparación de los significados y sentidos se ha tratado de analizar las similitudes y diferencias entre los casos, con el fin de explicar cómo y por qué se han desarrollado esos "modos de organización" de los procesos de acreditación institucional, a fin de levantar buenas prácticas.

Finalmente, la elección de las universidades se basa en tres aspectos principales. El primero, por su relevancia en el quehacer universitario del país y principalmente de la zona sur-austral ${ }^{7}$. En segundo lugar, porque al compartir una misma zona geográfica y pertenecer a plataformas transinstitucionales comunes ( $\mathrm{CRUCH}$ y la

7 La UACh nace en 1954, mientras que la UFRO y la ULagos nacen en los últimos 30 años (la UFRO en 1981 y la ULagos en 1993) como instituciones autónomas, pero como continuación de proyectos universitarios estatales anteriores. Asimismo, según el Ranking de América Economía 2012, la UACh aparece en $8^{\circ}$ lugar, la UFRO en $13^{\circ}$ lugar, y la ULagos ocupa el lugar 30 dentro de las 59 universidades autónomas del país. 
Red Universitaria Nacional REUNA) presentan ciertas condiciones que les son consustanciales a las tres, además de contar con un cierto grado de fraternidad expresado en vínculos institucionales y a nivel de autoridades y funcionarios.

El tercero de los aspectos contemplados y el más importante, se refiere a que estas tres universidades constituyen ejemplos de diferentes "modos de organización" de la acreditación: la Universidad Austral (UACh) funciona de manera descentralizada a través de una Oficina de Autoevaluación Institucional dependiente de la Vicerrectoría Académica, conformada por cuatro personas, de las cuales la mitad trabaja media jornada, y que tiene como tarea principal apoyar los procesos de autoevaluación de las distintas unidades. La Universidad de La Frontera (UFRO), por su parte, cuenta con una Dirección de Análisis y Desarrollo Institucional conformada por diez personas a tiempo completo, y que centraliza todo lo relativo al aseguramiento de la calidad: información y estudios para la planificación, la toma de decisiones y la formulación de políticas; formulación y actualización del Plan Estratégico; y procesos de acreditación. Finalmente, la Universidad de Los Lagos (ULagos) cuenta con una Dirección de Gestión y Aseguramiento de la Calidad dependiente de la Vicerrectoría de Planificación y Desarrollo, conformada por ocho personas a jornada completa, distribuidas en las tres sedes que posee la Universidad, y cuyo principal objetivo es promover una cultura de calidad institucional a partir de un rol de planificación, acompañamiento, asesoría y facilitación de procesos de calidad, dentro de los cuales se encuentran los procesos de autoevaluación y acreditación, tanto a nivel institucional como de programas.

\section{Análisis de la información}

\subsection{Tabla comparativa de aspectos formales ${ }^{8}$}

A continuación se presentan los elementos más destacados referidos a los aspectos formales de las instituciones consideradas para este estudio.

8 Toda la información fue proporcionada por las mismas universidades, con excepción del número de estudiantes y de JCE que se obtuvo del SIES. 
60 RADIOGRAFÍA DE LOS MODELOS DE ACREDITACIÓN: ORGANIZACIÓN, PROCESOS Y PRÁCTICAS. EL CASO DE LAS UNIVERSIDADES AUSTRAL DE CHILE, DE LA FRONTERA Y DE LOS LAGOS - J. P. Venables, J. Van Gastel

\subsubsection{Información institucional}

\begin{tabular}{l|c|c|c}
\hline & $\begin{array}{c}\text { Universidad Austral de } \\
\text { Chile }\end{array}$ & $\begin{array}{c}\text { Universidad de La } \\
\text { Frontera }\end{array}$ & \multicolumn{1}{c}{$\begin{array}{c}\text { Universidad de Los } \\
\text { Lagos }\end{array}$} \\
\hline $\begin{array}{l}\text { Año de fundación } \\
\text { y naturaleza } \\
\text { jurídica }\end{array}$ & $\begin{array}{l}\text { Se fundó en 1954. } \\
\text { Corporación de derecho } \\
\text { privado. }\end{array}$ & $\begin{array}{l}\text { Se fundó en 1981 lfusión } \\
\text { de UCH y UTE, sedes } \\
\text { Temuco). } \\
\text { Pública y estatal. }\end{array}$ & $\begin{array}{l}\text { Se fundó en 1993 lex } \\
\text { Instituto Profesional Los } \\
\text { Lagos, que nació de la } \\
\text { fusión de la UCH y la } \\
\text { UTE, sedes Osorno). } \\
\text { Pública y estatal. }\end{array}$ \\
\hline $\begin{array}{l}N^{\circ} \text { de } \\
\text { estudiantes } \\
\text { pregrado 2014 }\end{array}$ & 12.820 & 9.147 & 9.561 \\
\hline $\begin{array}{l}N^{\circ} \text { de } \\
\text { académicos } \\
\text { 2013 (JCE) }\end{array}$ & 838 & 330 & 339 \\
\hline $\begin{array}{l}\text { Número de años } \\
\text { de acreditación y } \\
\text { áreas }\end{array}$ & 6años (2009-2015) & $\begin{array}{l}\text { Todas las áreas. } \\
\text { Todas las áreas. }\end{array}$ & $\begin{array}{l}\text { 3 años (2013-2016) } \\
\text { Gestión Institucional, } \\
\text { Docencia de Pregrado y } \\
\text { Vinculación con el Medio. }\end{array}$ \\
\hline $\begin{array}{l}\text { Año último } \\
\text { proceso de } \\
\text { acreditación }\end{array}$ & 2009 & 2013 & 2013 \\
\hline
\end{tabular}




\subsubsection{Información unidades encargadas de conducir el proceso de autoevaluación}

\begin{tabular}{|c|c|c|c|}
\hline & $\begin{array}{c}\text { Universidad } \\
\text { Austral de Chile }\end{array}$ & $\begin{array}{c}\text { Universidad } \\
\text { de La Frontera }\end{array}$ & $\begin{array}{c}\text { Universidad } \\
\text { de Los Lagos }\end{array}$ \\
\hline $\begin{array}{l}\text { Año de creación y } \\
\text { nombre primera } \\
\text { unidad }\end{array}$ & $\begin{array}{l}\text { 1999. Oficina de } \\
\text { Autoevaluación } \\
\text { Institucional. }\end{array}$ & $\begin{array}{l}\text { 2003. Dirección de } \\
\text { Autoevaluación y } \\
\text { Acreditación. }\end{array}$ & $\begin{array}{l}\text { 2007. Unidad de } \\
\text { Aseguramiento de la } \\
\text { Calidad. }\end{array}$ \\
\hline $\begin{array}{l}\text { Año de creación } \\
\text { y nombre actual } \\
\text { Unidad }\end{array}$ & Sigue siendo la misma. & $\begin{array}{l}\text { 2008. Dirección de Análisis } \\
\text { y Desarrollo Institucional. }\end{array}$ & $\begin{array}{l}2010 \text { (2013 se formaliza). } \\
\text { Dirección de Gestión y } \\
\text { Aseguramiento de la } \\
\text { Calidad. }\end{array}$ \\
\hline $\begin{array}{l}\text { Objetivo principal } \\
\text { y/o misión de la } \\
\text { unidad }\end{array}$ & $\begin{array}{l}\text { Contribuir al desarrollo } \\
\text { de capacidades para } \\
\text { realizar autoevaluación en } \\
\text { todas las actividades de } \\
\text { la Universidad, mediante } \\
\text { prácticas de observación } \\
\text { rigurosa de la calidad, y } \\
\text { aplicar mejora continua } \\
\text { utilizando estándares de } \\
\text { comparación competitiva } \\
\text { de alto nivel. Convertir la } \\
\text { evaluación institucional en } \\
\text { un potente instrumento } \\
\text { para obtener diagnósticos } \\
\text { de la situación y } \\
\text { desempeño de las } \\
\text { funciones institucionales } \\
\text { y así fundamentar su } \\
\text { planificación. }\end{array}$ & $\begin{array}{l}\text { Contribuir al desarrollo } \\
\text { de una gestión } \\
\text { institucional basada } \\
\text { en el aseguramiento } \\
\text { de la calidad; proveer } \\
\text { información y estudios } \\
\text { de análisis para la } \\
\text { planificación, la toma } \\
\text { de decisiones y la } \\
\text { formulación de políticas; } \\
\text { coordinar la formulación } \\
\text { y actualización del Plan } \\
\text { Estratégico de Desarrollo } \\
\text { de la Institución; y } \\
\text { apoyar la formulación, } \\
\text { seguimiento y supervisión } \\
\text { del logro de objetivos de } \\
\text { proyectos de desarrollo } \\
\text { institucional. }\end{array}$ & $\begin{array}{l}\text { Proponer y promover } \\
\text { un sistema de gestión } \\
\text { y aseguramiento de } \\
\text { la calidad interna, } \\
\text { que favorezca los } \\
\text { procesos académicos } \\
\text { y administrativos del } \\
\text { quehacer universitario. } \\
\text { Promover la cultura } \\
\text { de la calidad y de la } \\
\text { autoevaluación, como } \\
\text { condición fundamental } \\
\text { para autorregular y } \\
\text { retroalimentar los } \\
\text { procesos académicos } \\
\text { y administrativos en la } \\
\text { institución. }\end{array}$ \\
\hline Subunidades & No. & $\begin{array}{l}\text { Estudio e información. } \\
\text { Evaluación y desarrollo } \\
\text { institucional. }\end{array}$ & $\begin{array}{l}\text { Unidad campus Puerto } \\
\text { Montt. } \\
\text { Unidad campus Santiago. }\end{array}$ \\
\hline $\begin{array}{l}\text { Número de } \\
\text { funcionarios, } \\
\text { perfiles y } \\
\text { jornadas }\end{array}$ & $\begin{array}{l}\text { Cuatro funcionarios (dos } \\
\text { con jornada completa y } \\
\text { dos con media jornada): } \\
\\
\text { jefe de Oficina, media } \\
\text { jornada. } \\
\text { Analista, jornada completa. } \\
\text { Profesional de apoyo, } \\
\text { jornada completa. } \\
\text { Secretaria jornada } \\
\text { completa (compartida con } \\
\text { otras unidades). }\end{array}$ & $\begin{array}{l}\text { Diez funcionarios (todos } \\
\text { con jornada completa): } \\
\text { director. } \\
\text { coordinador de Proyectos. } \\
\text { Ingeniero de Estudios. } \\
\text { Ingeniero de Planificación } \\
\text { y Gestión. } \\
\text { Dos ingenieros de Gestión } \\
\text { de Calidad. } \\
\text { Ingeniero de Análisis } \\
\text { Financiero. } \\
\text { Administrador financiero. } \\
\text { asistente financiero. } \\
\text { Secretaria administrativa. }\end{array}$ & $\begin{array}{l}\text { Ocho funcionarios (todos } \\
\text { con jornada completa): } \\
\text { directora. } \\
\text { Informático. } \\
\text { Sociólogo. } \\
\text { Coordinadora académica } \\
\text { de la Dirección. } \\
\text { Jefes de Gestión de } \\
\text { Aseguramiento de la } \\
\text { Calidad campus Puerto } \\
\text { Montt y Santiago. } \\
\text { Profesionales de apoyo } \\
\text { para cada campus. }\end{array}$ \\
\hline $\begin{array}{l}\text { Dependencia } \\
\text { intrainstitucional }\end{array}$ & Vicerrectoría Académica. & Rectoría. & $\begin{array}{l}\text { Vicerrectoría de } \\
\text { Planificación y Desarrollo }\end{array}$ \\
\hline $\begin{array}{l}\text { ¿Monitorea el } \\
\text { cumplimiento } \\
\text { de los planes de } \\
\text { mejora? }\end{array}$ & Sí. & Sí. & Sí. S S n \\
\hline
\end{tabular}


62 RADIOGRAFÍA DE LOS MODELOS DE ACREDITACIÓN: ORGANIZACIÓN, PROCESOS Y PRÁCTICAS. EL CASO DE LAS UNIVERSIDADES AUSTRAL DE CHILE, DE LA FRONTERA Y DE LOS LAGOS - J. P. Venables, J. Van Gastel

\subsection{Categorías de análisis}

A continuación se presenta una breve definición de cada categoría de análisis utilizada en la investigación, seguida de los principales resultados del análisis comparado de las tres universidades estudiadas ${ }^{9}$.

\subsubsection{El proceso histórico de su conformación}

Esta categoría de análisis tiene como finalidad comprender los "modos de organización" de los procesos de acreditación a través de una reconstrucción de la historia del desarrollo de los sistemas de autoevaluación al interior de cada universidad. La reconstrucción se enfoca en conocer cómo los actores clave en estos procesos de "traducción", cimentaron las bases para la conformación del "significado" -siempre inacabado, por cierto- que las políticas de aseguramiento de la calidad han ido adquiriendo en cada caso.

El principal objetivo de esta categoría es dar cuenta de cómo estas historias explican y/o justifican la estructura organizacional que hoy en día tienen las universidades en torno al aseguramiento de la calidad y la manera en que sus sistemas de autoevaluación funcionan.

\section{a. Análisis comparado Categoría A}

Uno de los aspectos que más llama la atención es que en las tres universidades los primeros encargados de estas unidades relevaron su propia experiencia en el extranjero y las buenas prácticas que recogieron de universidades europeas y estadounidenses como insumo fundamental para desarrollar un sistema de autoevaluación, o bien, para poder sistematizar en un primer momento los procesos internos de la universidad en relación con estos temas. En efecto, en la UFRO fue fundamental la experiencia que el primer director de Autoevaluación y Acreditación adquirió estudiando un doctorado vinculado con la construcción y transferencia de conocimiento en Estados Unidos. Asimismo, en la ULagos, el creador del Sistema de

9 Dadas las características de este artículo, se privilegió la exposición de los resultados por sobre el ejercicio comparativo. Para mayor información acerca de este, véase la investigación completa disponible en el sitio web de CNED (http://cned.cl/public/Secciones/ SeccionInvestigacion/investigacion_estudios_documentos.aspx). 
Aseguramiento de la Calidad hoy operativo fue el entonces vicerrector de Planificación y Desarrollo, quien había estudiado un doctorado y trabajado en distintas universidades suecas. En la UACh, por su parte, el primer jefe de la Oficina de Autoevaluación Institucional llegó a la Universidad por un proyecto de asistencia técnica y cooperación de la GTZ alemana, y tuvo como principal labor apoyar la confección de planes estratégicos, labor que se inspiró en lo realizado por la Universidad de Kassel, en Alemania.

Pero no sólo las experiencias extranjeras de los primeros encargados fueron importantes. También lo fue la obtención de apoyo e información a través de redes de universidades dentro Chile, y en particular del Centro Interuniversitario de Desarrollo (CINDA), como espacio para el intercambio de información, buenas prácticas y experiencias entre la red de universidades que lo conforma. En el caso de la ULagos y la UACh sigue siendo importante participar en estas instancias y, de hecho, la UACh lo releva como un aspecto importante dentro de su quehacer actual.

Vinculado con lo anterior, resulta destacable también que todas las unidades comienzan siendo pequeñas, operativas y con un perfil eminentemente técnico, que buscaba dar apoyo a ciertos procesos cuyos responsables eran otras unidades dentro de la universidad. A su vez, todas nacen como respuesta interna de las universidades a requerimientos externos. Vale decir, en los tres casos -aunque con distintos énfasis- se observan procesos de "traducción" de demandas provenientes del Estado, que obligaron a realizar modificaciones estructurales y de funcionamiento institucional, y que trajeron como resultado la adopción de ciertos "significados" particulares de calidad, autoevaluación, acreditación, autorregulación, planificación, mejora continua, etc. ${ }^{10}$

En este contexto de internalización de exigencias externas llama también la atención que en los tres casos se haya privilegiado -al menos en un comienzo- un enfoque menos vertical, que puso énfasis en los aspectos de mejoramiento y acompañamiento por sobre

10 Este tema lo retomamos en la tercera categoría de análisis. 
64 RADIOGRAFÍA DE LOS MODELOS DE ACREDITACIÓN: ORGANIZACIÓN, PROCESOS Y PRÁCTICAS. EL CASO DE LAS UNIVERSIDADES AUSTRAL DE CHILE, DE LA FRONTERA Y DE LOS LAGOS - J. P. Venables, J. Van Gastel

los de control y seguimiento ${ }^{11}$, en circunstancias que los mismos encargados señalaron que, en la práctica, el control y el seguimiento son inseparables del ejercicio de la autoevaluación y del aseguramiento de la calidad.

Lo anterior se relaciona, sin duda, con el temor y la subsecuente resistencia que estos procesos generaron en los académicos. Este aspecto fue destacado por la UFRO y la ULagos, lo que no quiere decir que no haya sucedido en la UACh. Puesto que toda imposición de sistemas de medición y control genera incertidumbre, y considerando que los académicos en las universidades tradicionales chilenas suelen contar con altos niveles de autonomía y poder sobre las decisiones institucionales, la mejor manera de hacerlo fue relevando las funciones de acompañamiento y apoyo de estas unidades, por sobre las de seguimiento y control.

Otro aspecto muy importante de destacar es que en los tres casos estas unidades comienzan sus funciones ligadas a la planificación estratégica. Incluso más, la destacan como uno de los primeros mecanismos de aseguramiento de la calidad impulsados en las universidades. Este aspecto tiene mucha relevancia a la luz de las tres funciones principales que suelen ir vinculadas con estas unidades, destacadas por Lemaitre et al. (2012): análisis institucional, planificación estratégica y aseguramiento de la calidad. Sin embargo, como veremos más adelante, no todas estas unidades continúan desarrollando funciones de planificación y análisis. El caso de la UACh es paradigmático en este sentido, pues si bien, como cuenta el primer jefe de la Oficina de Autoevaluación Institucional, en un comienzo su principal labor fue apoyar la confección de planes estratégicos, con el tiempo esa dejó de ser tarea de esta unidad. La Dirección de Gestión y Aseguramiento de la Calidad de la ULagos, por su parte, tampoco concentra esta tarea dentro de la unidad, pero participa en instancias colegiadas de planificación y análisis y, además, depende de la Vicerrectoría de Planificación y Desarrollo. La UFRO, en tanto, desde 2004, año en que se creó la Dirección de Aseguramiento de la Calidad y Desarrollo Institucional -antecesora de la actual Dirección

11 Este tema lo retomaremos en la última categoría de análisis. 
de Análisis y Desarrollo Institucional- concentra las tres funciones en esta unidad.

\subsubsection{La configuración, funciones y posición de la unidad dentro} de la estructura institucional

Esta categoría tiene relación con las importantes diferencias que se pueden observar en la materialización organizacional del Sistema Nacional de Aseguramiento de la Calidad entre las diversas universidades. En ese sentido, la formación de estas unidades ha surgido de un proceso de materialización de políticas y, por lo tanto, establece y consolida estas mismas políticas. Sin embargo, estas unidades, en tanto artefactos humanos, expresan ciertos significados, valoraciones y creencias (Yanow, 1996), no sólo en relación con el tema del mejoramiento de la calidad, sino también con el estatus institucional y con la obtención de recursos económicos asociados. En ese contexto, la posición que ocupa la unidad en la estructura organizacional, la configuración de su orgánica interna y las funciones que desempeña, son una expresión de "significado" que emana de este proceso de "traducción".

\section{a. Análisis comparado Categoría B}

Lo primero que llama la atención en relación con este proceso de "significación" y "traducción" es la diferencia en cuanto al alcance de estas unidades. En efecto, si comparamos en virtud de las tres funciones principales que se han ido incorporando como labores propias de estas unidades, es decir, planificación estratégica, análisis institucional y aseguramiento de la calidad (Lemaitre et al., 2012), es posible sostener, en términos generales, que mientras la Oficina de Autoevaluación Institucional de la UACh y la Dirección de Gestión y Aseguramiento de la Calidad de la ULagos han concentrado sus funciones en el aseguramiento de la calidad -y con ello en las distintas exigencias derivadas de la acreditación-, la Dirección de Análisis y Desarrollo Institucional de la UFRO concentra las tres funciones.

Ahora bien, haciendo un análisis más detallado, podemos observar que existen diferencias y similitudes entre las tres. Si bien tanto la Unidad de la ULagos como la de la UACh relevan mayormente 
las labores relacionadas con la acreditación dentro de la descripción de sus funciones (nueve de once funciones se vinculan con la acreditación en el caso de la ULagos, en tanto que en la UACh son ocho de las nueve funciones las relacionadas), el énfasis en una y otra es distinto. Mientras en la ULagos las labores de la dirección y el reconocimiento que tiene dentro de la comunidad universitaria se vincula directamente con temas de "calidad", lo que se expresa, por ejemplo, en que cuentan con una jefa de Gestión y Aseguramiento de la Calidad en la sede de Santiago donde no se dicta ninguna carrera de pregrado, o en el Sistema de Aseguramiento de la Calidad de la ULagos en el cual se inserta el quehacer de esta Dirección, en el caso de la UACh las labores de la Oficina han estado históricamente más vinculadas con la acreditación y, en particular, con el apoyo de los procesos de autoevaluación. El nombre de cada unidad -Dirección de Calidad y Oficina de Autoevaluación- es por sí solo expresión de aquello.

La descripción de funciones de la Dirección de Análisis y Desarrollo Institucional de la UFRO, por su parte, sólo destina dos de las cinco funciones a la evaluación o autoevaluación y ninguna directamente a la acreditación. Por supuesto, lo anterior no obsta para que en la práctica parte importante de sus labores se vincule con los procesos de acreditación, pero es una expresión del énfasis y alcance que estas unidades tienen en su operar.

Asimismo, la dependencia y la posición que estas unidades ocupan al interior de la estructura organizacional de sus respectivas universidades también entregan luces respecto del proceso de "traducción" y "significación" de las políticas estatales acerca del aseguramiento de la calidad. En efecto, la Dirección de Análisis y Desarrollo Institucional de la UFRO es la única que no depende de ninguna vicerrectoría sino lo hace directamente del Rector, lo que le entrega una posición estratégica y un grado de influencia en las decisiones universitarias que no tienen las otras dos unidades.

Lo anterior va de la mano con el hecho de que esta Dirección concentra una mayor cantidad de funciones y que estas tienen un carácter más estratégico y político en comparación con las unidades 
de la UACh y la ULagos. Esto se refleja también en que cuenta con casi cuatro veces más profesionales que la Oficina de Autoevaluación Institucional de la UACh y un tercio más que la Dirección de Gestión y Aseguramiento de la Calidad de la ULagos, y en que su presupuesto anual es quince veces superior al de la UACh y sesenta veces al de la ULagos. Ahora bien, estos presupuestos no son comparables sin considerar que en el caso de la UFRO -dado que no depende de ninguna vicerrectoría- la Dirección maneja como centro de costo independiente las remuneraciones de sus funcionarios, mientras que en el caso de la ULagos y la UACh las remuneraciones no forman parte del presupuesto de la unidad. De todos modos, haciendo un ejercicio aproximado de calcular el costo de estas unidades sumando las remuneraciones (se debe considerar que en ambos casos los encargados reciben una asignación por su función, pero sus remuneraciones son costeadas por sus respectivas unidades académicas), el presupuesto de la Dirección de Análisis y Desarrollo Institucional de la UFRO sigue siendo, al menos, cinco veces mayor.

Pero la dificultad de comparar los presupuestos que manejan estas unidades se complejiza aún más si se consideran los gastos asociados a los procesos de acreditación con agencias o con la CNA. La misma directora de la ULagos comentó que algunos colegas creían que esa unidad "era rica", puesto que estos recursos se asociaban al centro de costos de su Dirección, cuestión que no sucede en la UACh. Esto permite relevar otra característica importante, que dice relación con la dependencia del área de gestión o académica del aseguramiento de la calidad. En efecto, tanto en la ULagos como en la UFRO los temas de aseguramiento de la calidad se manejan con un énfasis en la gestión, lo que se expresa claramente en su dependencia jerárquica de la Vicerrectoría de Planificación y Desarrollo y de Rectoría, respectivamente. Mientras que en el caso de la UACh, la Oficina depende de la Vicerrectoría Académica y su labor se entiende fundamentalmente como apoyo al quehacer académico de la Universidad. Todo esto, por supuesto, está también vinculado con las funciones que desarrolla la unidad, puesto que en el caso de la UFRO y la ULagos está, en distintos niveles, asociada con la planificación estratégica, lo que no sucede en la UACh. 
Dicho lo anterior, si bien el que sólo en la UACh esta unidad no tenga rango de dirección haría pensar que los casos de la ULagos y la UFRO son más parecidos entre sí en lo que se refiere a configuración, funciones y posición en la estructura organizacional, lo cierto es que se observa mayor confluencia entre las unidades de la ULagos y la UACh, toda vez que ambas cumplen un rol más técnico que estratégico, y ciertamente menos político que la unidad de la UFRO.

Finalmente, es importante señalar que las tres unidades parecen ser bien evaluadas por académicos (de acuerdo con lo señalado por los entrevistados) y por las autoridades superiores, lo que, en parte, da cuenta del éxito alcanzado por las tres universidades en el proceso de "traducción" y "significación" de las políticas de aseguramiento de la calidad emanadas desde el Estado, toda vez que lograron ser percibidas como una unidad de apoyo más que de control ${ }^{12}$.

\subsubsection{El significado de una cultura de autoevaluación}

Todas las universidades destacan como uno de los objetivos principales para el aseguramiento de la calidad, desarrollar o generar una "cultura" de evaluación, autoevaluación, calidad o autorregulación. Esta categoría explora el "significado" de "cultura" en cada universidad, destacando su importancia, cómo han intentado desarrollarla y qué resultados han obtenido; poniendo especial énfasis en la manera en que estas unidades han implementado mecanismos de control y/o autocontrol.

\section{a. Análisis comparado Categoría C}

Lo primero que se debe destacar es que, en los tres casos, el "significado" de cultura siempre está relacionado con otro concepto, es decir, se habla de una "cultura de" o de una "cultura para", lo que lo vuelve un término funcional cuyo "significado" solo cobra sentido al estar asociado con otra cosa. En efecto, vimos que en los tres casos la noción con la cual se vincula la cultura varía: en la UFRO se habla de una cultura de evaluación y autoevaluación, mientras que tanto en la ULagos como en la UACh se hace referencia a una cultura de

12 Este tema lo retomamos en la siguiente categoría de análisis. 
calidad, con la salvedad de que en esta última también se habla de cultura de autorregulación y de autoevaluación.

Sin perjuicio de lo anterior es posible observar que, finalmente, no existe tanta diferencia en cómo comprenden esta cultura. De hecho, las tres universidades señalan que, en última instancia, lo que entienden por cultura de calidad o autoevaluación tiene relación con la internalización de una cierta "actitud" por parte de todos los miembros y unidades universitarias, que exprese el entendimiento cabal de que el aseguramiento de la calidad y la mejora continua de la institución no depende de la gestión de estas unidades, sino de la adopción de esta actitud crítica, autoevaluativa y autorreguladora, por parte de cada miembro y unidad.

En definitiva, existe una doble concepción de la cultura de autoevaluación o de calidad, pues, por un lado se han tratado de instalar sistemas burocráticos de control, mientras que por otro, normas de trabajo y prácticas de autocontrol. En efecto, estos sistemas exigen contar con reglas de seguimiento y funcionan a través de documentos, formularios, reuniones formales y un orden estandarizado de acciones que van conformando la organización burocrática en el sentido descrito por Weber (1997). Pero, al mismo tiempo, la idea de cultura de autoevaluación y/o calidad tiene un fuerte componente de accountability, concepto que combina la idea de rendición de cuentas -vinculada con la eficiencia y la eficacia económica- y el razonamiento moral -relacionado con la idea de buenas prácticas- (Strathern, 2004). Por lo tanto, se requiere la creación de mecanismos donde antes no existían, pero la retórica que los acompaña suele ser la de ayudar (monitoreo) a que las personas se ayuden a sí mismas (monitor), incluyendo la ayuda para que se acostumbren a esta nueva 'cultura' (Strathern, 2004, p. $4^{13}$ ).

Esta última idea está vinculada también con las "tecnologías del yo" (technologies of the self) o de "autocontrol" (Foucault, 1979), es decir, se trata de la internalización individual de normas de trabajo (Kunda, 1992).

13 Traducción de los autores. 
70 RADIOGRAFÍA DE LOS MODELOS DE ACREDITACIÓN: ORGANIZACIÓN, PROCESOS Y PRÁCTICAS. EL CASO DE LAS UNIVERSIDADES AUSTRAL DE CHILE, DE LA FRONTERA Y DE LOS LAGOS - J. P. Venables, J. Van Gastel

Este doble "significado" de cultura de autoevaluación y/o calidad es consustancial a las tres universidades. En consecuencia, las diferencias entre ellas tienen que ver con una cuestión de grado o énfasis, más que de orientación.

Ahora bien, también es posible observar diferencias en la manera en que las universidades han tratado de instalar o desarrollar esta cultura. Vale decir, las diferencias se observan en las acciones y prácticas llevadas a cabo por las unidades encargadas de estos procesos, más que en un "significado" particular de cultura. Es así como, tanto en la UFRO como en la UACh, el desarrollo de esta cultura parece estar aparejado con un "modo de organización" de los procesos de autoevaluación que entrega a las mismas unidades la responsabilidad de desarrollarlos y escribir los informes (Kunda, 1992; Yanow, 1996). En el caso de la UFRO esto es más claro aún, producto del trato y el lenguaje de servicio al cliente con que esta unidad se relaciona con las unidades académicas (a quienes llaman "clientes"), cuestión que también se refleja en la "arquitectura" de su oficina.

Incluso más, el que la Dirección de Análisis y Desarrollo Institucional de la UFRO y la Oficina de Autoevaluación Institucional de la UACh hayan sido sistemáticamente explícitas en definirse como unidades técnicas de apoyo apunta en la misma dirección, toda vez que ello hace referencia a que esta cultura debe ser una práctica internalizada por todas las unidades y miembros de la institución, reduciendo su labor como unidades a apoyar técnicamente procesos liderados en otro lado. En palabras simples, estas unidades no escriben los informes ni influyen en sus contenidos, sino más bien entregan orientaciones técnicas.

En el caso de la Dirección de Gestión y Aseguramiento de la Calidad de la ULagos, el desarrollo de esta cultura ha estado asociado a prácticas un tanto más directivas, que han puesto el acento en tratar de instalar el tema de la calidad en los académicos a través de la elaboración de documentos y mecanismos de control (planes de calidad). En efecto, la ULagos primero se centró en concientizar a sus académicos respecto de la importancia de imprimir un "sello de calidad" en su trabajo, lo que se definió en términos de "el 
cumplimiento de los propósitos y fines declarados por la institución". Asimismo, se implementó un sistema de control de calidad que evalúa el desarrollo docente de los académicos y, además, la Dirección adquirió un rol más protagónico en la escritura de los informes de autoevaluación de las carreras y programas, así como en la revisión de los planes de calidad, siendo la instancia que les da el visto bueno final.

Sin embargo, lo anterior no obsta para que también en la ULagos sean las carreras y los programas los encargados de escribir sus informes. Las diferencias son de énfasis más que de orientación. Es más, el hecho de que la directora haga referencia a la cultura de calidad y autoevaluación como una cierta actitud que debe estar en todos los miembros de la institución, refuerza esta idea.

Finalmente, es importante abordar la vinculación entre la "cultura de calidad" y la "cultura de autoevaluación", que se utiliza indistintamente en aquellas instituciones donde se habla de ambos (ULagos y UACh). No obstante, se podría sostener que la cultura de autoevaluación sería el medio a través del cual alcanzar el fin que estaría dado por la cultura de calidad. Pero lo más interesante de rescatar es que, al parecer, en las tres universidades se está comprendiendo preferentemente la calidad en su dimensión de "consistencia interna", es decir, como la "adecuación a los propósitos declarados" según Lester (en Lemaitre et al., 2012). Para ello, lo más importante sería contar con una cultura de autoevaluación que se erija como el medio a través del cual las distintas unidades y miembros de la institución se revisen permanentemente a sí mismos y a su quehacer. Pero, ¿dónde queda en esta definición el medio externo? De lo que se pudo desprender del análisis, la incorporación de la "consistencia externa" dentro de las políticas de aseguramiento de la calidad de las universidades, es un desafío por considerar.

\subsubsection{Cambios recientes. Desafíos y perspectivas estratégicas, políticas y técnicas}

Esta categoría se adentra en el recorrido de modificaciones estructurales y funcionales recientes que las unidades encargadas han experimentado y, al mismo tiempo, indaga acerca de sus proyecciones y desafíos futuros. Todo ello, observado bajo la distinción entre 
aquellas unidades que privilegian una función más estratégica y/o política, o bien, un rol más técnico.

\section{a. Análisis comparado Categoría D}

Lo primero que debe destacarse es que las tres unidades se han mantenido con un alto nivel de estabilidad en el tiempo. La Oficina de Autoevaluación Institucional de la UACh es la más antigua de las tres y no ha realizado ningún cambio formal de sus funciones, configuración organizacional ni posición dentro de la estructura universitaria. Más aún, desde el año 2002 que cuenta prácticamente con el mismo personal. En una línea similar, la Dirección de Análisis y Desarrollo Institucional de la UFRO ha mantenido la misma línea que sus unidades predecesoras, y desde 2008 que no presenta cambios formales de función ni posición organizacional y sólo ha incorporado un par de profesionales al equipo.

La Dirección de Gestión y Aseguramiento de la Calidad de la ULagos, por su parte, es la que más cambios ha sufrido desde su creación en 2007. Sin embargo, si bien el cambio más reciente data de 2013 y tiene que ver con su posicionamiento organizacional (pasó a ser Dirección y a ocupar un nivel estratégico), desde 2010 que en la práctica se le consideraba así. Asimismo, en 2011 se incorpora la actual directora y, además, las funciones de la unidad no se han modificado sustancialmente, lo que al menos permite hablar de una estabilización en los últimos años.

En segundo lugar, es importante recalcar que, siguiendo a Latour (1993), no existe una separación clara y concreta entre las labores estratégico-políticas y técnicas y, por tanto, el ejercicio de dilucidar el énfasis de cada unidad no responde a una visión dicotómica ni antagonista sobre ambas. Por el contrario, se entiende como propio de este tipo de unidades la tensión entre el carácter técnico y político en el desempeño de sus labores; tensión que no se supera, sino más bien se maneja de uno u otro modo.

Dicho lo anterior, y vinculando los cambios recientes de función, configuración organizacional y posicionamiento dentro de la estructura universitaria con los desafíos identificados para cada una 
de estas unidades, pueden observarse distintas situaciones. En efecto, la Dirección de Gestión y Aseguramiento de la Calidad de la ULagos se encuentra inserta dentro de un proceso de transformación en marcha, vinculado con las distintas acciones que persiguen potenciar la Vicerrectoría de Planificación y Desarrollo y sus unidades, lo que redundaría en una ampliación de las funciones de la Dirección agregando mayor análisis y entregándole mayor peso político a la Vicerrectoría y sus unidades. Esto se refleja en lo señalado por el rector, la directora y otros actores y, a su vez, se complementa con el deseo de transitar hacia una versión 2.0 de su directora, lo que permitiría desburocratizar los temas relacionados con el aseguramiento de la calidad.

La Oficina de Autoevaluación Institucional de la UACh, por su parte, no ha comenzado todavía un proceso de cambio, pero parece contar con un diagnóstico compartido respecto de la necesidad de elevar el posicionamiento dentro de la estructura organizacional de la unidad, pasando de Oficina a Dirección. Asimismo, es compartido que este cambio no implicaría asumir más funciones, puesto que tanto la planificación estratégica como el análisis institucional se encuentran afincados en otras unidades que, por lo demás, dependen de otra vicerrectoría. Por lo tanto, este cambio de posicionamiento iría en la línea de potenciar el rol técnico de la unidad, pero no de entregarle mayor peso político ni poder de injerencia en las decisiones.

La Dirección de Análisis y Desarrollo Institucional de la UFRO también presenta ciertos desafíos, pero difiere de la UACh y la ULagos en que no existe total consenso respecto de cuáles son éstos, lo que queda graficado en la figura de la "controversia". En efecto, como señala Latour (2008), una controversia se refiere a un proyecto, un objeto o un tema que aún no está estabilizado. Ello no implica necesariamente que se esté frente a una disputa ni tampoco que sea un tema politizado, sino que los actores no están de acuerdo, o bien, están de acuerdo de su desacuerdo (Latour, 2008). El hecho de que el rector manifieste su deseo de que esta dirección se transforme en el "cerebro pensante" de la Universidad, mientras su director considere que esa labor no le corresponde en exclusiva a esta unidad, sino más bien esta debe mantener su rol técnico, haciendo descansar esta labor 
en espacios colectivos como los cuerpos colegiados superiores, da cuenta de que están en una controversia. El que todavía no sea tema de agenda refuerza esta idea.

Finalmente, parece existir un cierto consenso entre los encargados en que una buena práctica en relación con estas unidades es que, en la medida que se han ido consolidando, la función que desarrollan "deja de ser meramente técnica con respecto de la conducción y desarrollo de procesos de acreditación. Si bien esta función sigue existiendo, se encuentra ubicada en otro nivel [...] o bien forma parte de la dirección, siendo una de sus funciones, pero no la principal" (Lemaitre et al., 2012, p. 42). Este es, sin duda, el caso de la Dirección de Análisis y Desarrollo Institucional de la UFRO, que desde un comienzo se planteó con estas características. Asimismo, la Dirección de Gestión y Aseguramiento de la Calidad de la ULagos parece estar tendiendo a una transformación en ese sentido.

La Oficina de Autoevaluación Institucional de la UACh, por su parte, no tiene proyectado crecer hacia la integración de funciones cuestión que, por cierto, no responde sólo a los intereses de la unidad, sino a la manera cómo la Universidad en su conjunto, y principalmente sus autoridades superiores, conciben los procesos de aseguramiento de la calidad. Sin perjuicio de lo anterior, llama la atención que pese a este modelo de gestión de aseguramiento de la calidad, la UACh presente los mejores resultados en términos de años y áreas de acreditación.

\section{Conclusiones}

La perspectiva teórico-conceptual utilizada-comprender el proceso de instalación de mecanismos de aseguramiento de la calidad al interior de las universidades como un proceso de "traducción" y generación de "significado"- permitió revisar empíricamente las diferencias y semejanzas en los "modos de organización" de los procesos de autoevaluación con miras a la acreditación institucional -y de procesos asociados: monitoreo de planes de mejora, aseguramiento de la calidad, análisis institucional y, en algunos casos, planificación estratégica-, así como también profundizar en las características particulares de cada una de las unidades encargadas de desarrollarlos. 
En este contexto, lo primero que cabría destacar es que las tres universidades investigadas han concentrado las funciones de "traducción" y "significación" en unidades especializadas, cuya labor principal es -en la práctica, independientemente de sus declaraciones formales- apoyar el desarrollo de los procesos de autoevaluación al interior de la institución. En efecto, la alta demanda que esta labor ha ido adquiriendo con el tiempo al interior de las IES, producto de la legitimidad social que ha alcanzado el sistema de acreditación -pese a sus múltiples problemas- y a la obtención de recursos económicos asociados, ha hecho que estas unidades se vuelvan imprescindibles y cada vez más requeridas estratégicamente.

Asimismo, se destaca la variabilidad de "significados" que este proceso de "traducción" trajo aparejado en las universidades investigadas, lo que puede observarse en las particularidades históricas asociadas a la formación de estas unidades y en la diversidad de funciones asumidas, así como en su composición profesional y en la posición que ocupan dentro de la estructura organizacional, entre otras. De este modo, una diferencia que pareciera meramente procedimental, como lo es la dependencia intrainstitucional de estas unidades, expresa claramente el énfasis y la orientación que cada universidad ha querido dar a este proceso de "traducción".

Sobre este mismo punto, es importante destacar que el proceso de "traducción" y generación de "significados" es, siempre, una operación sociolingüística interpretable de diversas formas por los distintos actores que forman parte del grupo social que la sustenta (Latour, 1996; Yanow, 2013) -lo que no obsta para que existan "horizontes de significado compartido"-, así como también un "dispositivo histórico" que, como tal, está en un proceso permanente de revisión y transformación (Koselleck, 1993). En virtud de ello, tomando y adaptando lo señalado por Latour (1996) respecto de este contexto específico, se puede sostener que todo proceso de "traducción" genera, simultáneamente, mecanismos de "estabilización" de los "significados" generados. En este caso, los mecanismos de "estabilización" utilizados por estas unidades encuentran una buena plataforma de materialización en la "cultura". El caso de la ULagos es el más paradigmático al respecto, toda vez 
que el Sistema de Aseguramiento de la Calidad creado para uso interno tiene entre sus prácticas más difundidas la generación de documentos, folletería, manuales, entre otros, vinculados con la calidad. La UFRO, por su parte, tiene en el diseño y utilización de los espacios de la oficina de la Dirección de Análisis y Desarrollo Institucional, así como en el lenguaje de prestación de servicios a "clientes" utilizado por sus funcionarios, una muestra importante de dispositivos culturales utilizados como "mecanismos de estabilización de significados". La Oficina de Autoevaluación Institucional de la UACh, finalmente, ha privilegiado el trabajo "en terreno", acercándose a las distintas unidades que así lo requieran para difundir una cultura de autorregulación, al mismo tiempo que diseñó un modelo de autoevaluación para ser aplicado por las unidades de gestión dentro de la Universidad.

Ahora bien, no obstante las características particulares de cada unidad en el manejo de estos "mecanismos de estabilización de significados", es consustancial a todas el uso de un cierto lenguaje y la producción de documentos que les den soporte. Es así como las tres universidades han generado manuales para la autoevaluación de carreras y programas, así como otros documentos que definen las etapas de los procesos, explicitan la definición de calidad de la universidad, o "traducen" en códigos internos los términos de referencia de la CNA.

Otro aspecto importante de destacar es que las tres unidades viven de manera reconocida la tensión de ser unidades técnicas y estratégicas en forma simultánea. Asimismo, también llama la atención que en los tres casos se reivindique la importancia de la labor técnica desempeñada, principalmente por la independencia política que entrega para el trabajo con las distintas unidades académicas, así como también por la posibilidad de poner énfasis en el acompañamiento por sobre el control.

En cuanto a cómo influyen estos distintos "modos de organización" de la acreditación en los resultados obtenidos, coincidimos con Lemaitre et al. (2012) acerca de la dificultad de establecer relaciones causales y explicativas entre ciertas prácticas 
y el número de años de acreditación. En consecuencia, manejamos dos hipótesis que cuentan con evidencia empírica que las sustenta y que, a primera vista, aparecen como contradictorias, aun cuando lo son sólo en sus resultados y no en sus contenidos.

La primera tiene relación con que el aseguramiento de la calidad debe comprenderse sistémicamente y, como tal, sería multifactorial en cuanto a sus fuentes de influencia. Por lo tanto, para alcanzar mejores resultados en la acreditación no bastaría sólo con la creación de unidades o estructuras internas que se hagan cargo de llevar adelante los procesos de acreditación y/o aseguramiento de la calidad, ni con perfeccionar su labor técnica con más recursos humanos y financieros, puesto que tendrían la misma influencia otros factores menos manejables centralizadamente, como el compromiso y el grado de participación de los académicos, la autonomía con que toman las decisiones las distintas unidades, la cultura institucional, la historia de la universidad, el medio en el cual se inserta, etc. En consecuencia, el resultado dependería más de la institución en su totalidad, incluyendo aspectos que escapan a la estandarización y al control.

El caso de la UACh sería un ejemplo paradigmático de esta situación, pues es una de las universidades del país con mejores resultados de acreditación, y la unidad encargada de desarrollar y orientar estos procesos es una oficina pequeña, con bajo presupuesto y con un jefe académico que, en lo formal, dedica media jornada a esta labor. En el caso de esta hipótesis, los buenos resultados de la UACh se explicarían por factores institucionales que escapan al control de esta u otra unidad.

La segunda hipótesis es más aventurada, por el hecho de contar con menos evidencia empírica que la sustente; no obstante, no por eso es menos plausible, y dice relación con que los resultados de la acreditación no tienen necesariamente una vinculación con los "modos de organización" de los procesos de aseguramiento de la calidad al interior de las IES, o al menos no en todos los casos. De ser así, los resultados de acreditación estarían perpetuando lógicas establecidas, al entregar años de acreditación en función del prestigio institucional y no del trabajo en torno al aseguramiento de la calidad. 
En este caso, la CNA no estaría haciendo una buena lectura de los desarrollos sistemáticos en torno a estos procesos que algunas IES han alcanzado, implementando sistemas integrales e integrados para el aseguramiento de la calidad. El caso de la ULagos daría cuenta de esta hipótesis pues, pese a contar con evidencia empírica respecto del importante avance en la instalación de mecanismos de aseguramiento de la calidad, los problemas que arrastra su historia de expansión no controlada seguirían influyendo en los resultados obtenidos.

De cualquier modo, resulta innegable el efecto positivo que ha traído la creación de unidades especializadas en aseguramiento de la calidad para el conjunto de IES.

Finalmente, se hace necesario mencionar las principales buenas prácticas identificadas a partir del análisis de la evidencia empírica obtenida, así como también algunos desafíos y debates. Las buenas prácticas se pueden organizar en cuatro puntos:

1. Se puede desprender que toda buena práctica depende del contexto organizacional y, por tanto, no es una buena práctica tomar sistemas o modelos creados en otro contexto y aplicarlas sin más. En este caso, las tres universidades investigadas destacan por haber asumido adecuadamente este proceso de "traducción" y haberse hecho cargo de la generación de "significados".

2. Más importante que contar con un sistema estructurado e integral de aseguramiento de la calidad, es que las distintas acciones tomadas en relación con estas temáticas sean coherentes entre sí y tengan un correlato uniforme al interior de la universidad.

3- Parece ser una buena práctica que estas unidades tiendan a concentrar las tres tareas vinculadas con el aseguramiento de la calidad: planificación estratégica, análisis institucional y autoevaluación. Le entrega mayor autonomía al trabajo de aseguramiento de la calidad, al mismo tiempo que congrega aspectos técnicos y político-estratégicos, ayudando a disminuir esta tensión inherente y volviéndolas unidades más complejas. Además, porque se pudo observar que los procesos de autoevaluación están intrínsecamente vinculados con la generación de indicadores y análisis institucional, al mismo tiempo que la generación de planes de mejora asociados a estos 
procesos y su respectivo monitoreo están hermanados con el ejercicio de prospección institucional propio de la planificación estratégica. Finalmente, es posible hacer un uso más eficiente de los recursos financieros, humanos e institucionales, vinculando la ejecución de los procesos de planificación y autoevaluación.

4. Uno de los problemas que se han suscitado con la instalación de estos procesos tiene relación con la resistencia que ha surgido de parte de los académicos. Pero esta no se debe sólo a la implementación de mecanismos de control, sino también a que implica más trabajo burocrático y de gestión. En consecuencia, parece una buena práctica que estas unidades realicen un proceso de desburocratización, que impida agobiar a los académicos con tareas de seguimiento y control que los desvíen de su función principal. Los cambios que la directora de Gestión y Aseguramiento de la Calidad de la ULagos quiere implementar, basados en tres principios tomados de AQU Catalunya -no debe tomar mucho tiempo, debe ser de fácil acceso y completación, y deben sentir que les sirve-, parecen apuntar en esa dirección.

Respecto de los desafíos, es posible destacar dos que, además, pueden ser extrapolables a todo el sistema. El primero, tiene que ver con que las tres unidades privilegian una visión de la calidad -y por ende del aseguramiento de la calidad- que presenta los inconvenientes de las definiciones que Lester (en Lemaitre et al., 2012) llama fitness for purpose (ajuste a los propósitos). En efecto, no fue posible observar que ninguna de las universidades relevara el papel de la retroalimentación con el medio externo como insumo permanente y estructurado para sus sistemas de aseguramiento de la calidad, más allá de declaraciones de intenciones. En este sentido, el desafío sería transitar hacia una "significación" en la línea de lo que Lester llama fitness of purpose (ajuste de los propósitos), asumiendo como fundamental las exigencias pertinentes del medio externo.

El segundo y último desafío dice relación con algo que parece una paradoja: las unidades encargadas del aseguramiento de la calidad no suelen autoevaluarse. Asimismo, tampoco suelen evaluar los sistemas mismos de autoevaluación y aseguramiento de la calidad. En el caso de las universidades investigadas, tanto la UFRO como 
80 RADIOGRAFÍA DE LOS MODELOS DE ACREDITACIÓN: ORGANIZACIÓN, PROCESOS Y PRÁCTICAS. EL CASO DE LAS UNIVERSIDADES AUSTRAL DE CHILE, DE LA FRONTERA Y DE LOS LAGOS - J. P. Venables, J. Van Gastel

la UACh señalaron que lo hacen como una práctica permanente, no formalizada, dentro de su quehacer cotidiano, mientras que la ULagos destacó hacer evaluación de sus funcionarios, aunque con descripciones de cargo no formalizadas.

\section{Bibliografía}

Almonacid, C., Luzón, A. y Torres, M. (2008). Cuasi mercado educacional en Chile: el discurso de los tomadores de decisión. Archivos Analíticos de Políticas Educativas, 16(8), 1-47.

Arata, A. y Rodríguez E. (2009). Desafíos y perspectivas de la dirección estratégica de las instituciones universitarias. Santiago: Ediciones CNA-Chile.

Cruz-Coke, R. (2004). Evolución de las universidades chilenas 1981-2004. Revista Médica de Chile, 132(12), 1.543-1.549.

Foucault, M. (1979). Discipline and punish: the birth of the prison. Londres: Penguin.

Gainza, A. (2006). La entrevista individual en profundidad. En M. Canales (coord.), Metodologías de investigación social. Introducción a los oficios (pp. 219-263). Santiago: LOM Ediciones.

IPSOS (2010). Estudio exploratorio sobre efectos de la acreditación institucional en la calidad de la educación superior en Chile. Recuperado de http:// www.cnachile.cl/wp-content/uploads/2010/07/INFORME_FINAL.pdf

IPSOSb (2010). Informe ejecutivo. Estudio exploratorio sobre efectos de la acreditación institucional en la calidad de la educación superior en Chile. Recuperado de http://www.cnachile.cl/wp-content/uploads/2010/07/ INFORME_FINAL.pdf.

Koselleck, R. (1993). Futuro pasado. Para una semántica de los tiempos históricos. Barcelona: Paidós.

Kunda, G. (1992). Engineering culture: control and commitment in a high-tech corporation. PA: Temple University Press.

Laclau, E. (2002). Misticismo, retórica y política. México: Fondo de Cultura Económica.

Latour, B. (1993). We have never been modern. Cambridge: Harvard University Press.

Latour, B. (1996 [2002]). Aramis, or the love of technology. Cambridge: Harvard University Press.

Latour, B. (2008). Reensamblar lo social. Buenos Aires: Edición Manantial. 
Law, J. (1994). Organizing modernity. Cambridge: Blackwell Publisher.

Lemaitre, M., Maturana, M., Zenteno, E. y Alvarado, A. (2012). Cambios en la gestión institucional en universidades, a partir de la implementación del Sistema Nacional de Aseguramiento de la Calidad: la experiencia chilena. Revista Calidad en la Educación, 36 (julio), 21-52.

Matear, A. (2007). Tensions between state and market in Chile: educational policy and culture. European Review of Latin American and Caribbean Studies [Revista Europea de Estudios Latinoamericanos y del Caribe], 83, 61-82.

Mosse, D. (2004). Is good policy unimplementable? Reflections on the Ethnography of aid policy and practice. Development and change, 35(4), 639-671.

Morgan, G. (2006 [1986]). Images of organization. Thousand Oaks, CA: Sage.

Yanow, D. (1996). How does a policy mean? Interpreting policy and organizational actions. Washington DC: Georgetown University Press.

Yanow, D. (2014). Interpretive analysis and comparative research. En I. Engeli \& C. Rothmayr (Eds.), Comparative policy studies: Conceptual and methodological challenges (pp. 131-159). Palgrave Macmillan.

Yin, R. (1994). Case study research: Design and methods. California: Sage.

Schatzki, T. R. (2001). Introduction: practice theory. En T.R. Schatzki, K. Knorr Cetina \& E. Von Savigny (Eds.), The practice turn in contemporary theory (pp. 10-23). Londres: Routledge.

Sepúlveda, C. (2011). Crisis de la educación superior chilena. Revista Chilena de Pediatría, 82(6), 483-484.

Shore, C. \& Wright, S. (2004 [2000]). Coercive accountability. The rise of audit culture in higher education. En M. Strathern (Ed.), Audit cultures. Anthropological studies in accountability, ethics and the academy (pp. 5789). Londres: Routledge.

Strathern, M. (2004). Introduction: New accountabilities. Audit cultures. Anthropological studies in accountability, ethics, and the academy (pp. 1-18). Londres: Routledge.

Weber, M. (1997). Esencia, supuestos y desarrollo de la dominación burocrática. En M. Weber (Autor), Economía y Sociedad. México: Fondo de Cultura Económica.

Recibido: 1/10/2014

Aceptado: 13/11/2014 